\title{
The Overlaps and Boundaries Between Service Design and User Experience Design
}

\author{
Virpi Roto \\ Aalto University \\ virpi.roto@aalto.fi \\ Effie Lai-Chong Law \\ University of Leicester \\ lcl9@leicester.ac.uk
}

\author{
Jung-Joo Lee \\ National University of Singapore \\ jjlee@nus.edu.sg \\ John Zimmerman \\ Carnegie Mellon University \\ johnz@andrew.cmu.edu
}

\begin{abstract}
The ongoing transition within tech industry towards holistic service journey orchestration pushes user experience design (UXD) closer to service design (SD). At the same time, new digital channels for traditional services pushes SD closer to UXD. The increasing interplay between UXD and SD can cause confusion about their relationship, boundaries, and responsibilities. Little research has examined perceptions of these aspects in industry and academia. We surveyed 197 UXD and SD practitioners and academics about their understanding of the overlaps and boundaries between the two design practices. Our findings reveal several conflicting views around the scope of UXD and SD work, the subsumptive relation between UXD and SD, and the theoretical roots and methods each draws upon. We suggest constructive ways for the two design practices to complement each other. Our study shows that UX work is changing, and understanding this change is important to maintain the relevance of UX research.
\end{abstract}

\section{CCS CONCEPTS}

- Human-Centered Computing $\rightarrow \mathrm{HCI}$ design and evaluation methods.

\section{KEYWORDS}

Service Design, User Experience Design, Design Practice, Survey

\section{ACM Reference Format:}

Virpi Roto, Jung-Joo Lee, Effie Lai-Chong Law, and John Zimmerman. 2021. The Overlaps and Boundaries Between Service Design and User Experience Design. In Designing Interactive Systems Conference 2021 (DIS '21), June 28-July 02, 2021, Virtual Event, USA. ACM, New York, NY, USA, 12 pages. https://doi.org/10.1145/3461778.3462058

\section{INTRODUCTION}

User Experience Design (UXD) and Service Design (SD) originate from different disciplinary backgrounds - UXD from HumanComputer Interaction (HCI) and SD from operations management

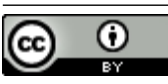

This work is licensed under a Creative Commons Attribution International 4.0 License.

DIS '21, fune 28- July 02, 2021, Virtual Event, USA

(c) 2021 Copyright held by the owner/author(s).

ACM ISBN 978-1-4503-8476-6/21/07.

https://doi.org/10.1145/3461778.3462058 and marketing. They both share the focus on user/customer experience, they often use the same methods (e.g., personas, customer journey maps, iterative prototyping), and they often overlap on projects. On the one hand, the increasing use of digital channels in service delivery requires SD to pay more attention to digital services and UX considerations, and it appears to move SD closer to UXD [17],[34]. On the other hand, the web and high speed internet helped transition of the software industry towards offering services instead of selling software products. This transition requires UXD to pay more attention to the distinction between users and customers, and to unfolding journey of users and customers. It moves UXD closer to SD [1],[27]. These two design practices have become so overlapping that it can sometimes be difficult to tell from job ads if a company wants a service designer, a UX designer, or someone who can do both [33].

This ongoing transformation in UX practice has led to some confusion around how UXD and SD should collaborate and coordinate in industry projects, and the research-practice gap in the area of UXD and SD seems to be widening. While some researchers have suggested that UXD might benefit from taking a service perspective [8], [14], studies at the intersection of UXD and SD are still rare [38],[20]. If UXD and SD practice are moving closer together, the research communities might benefit from coming closer as well. Today, they remain far apart. For example, few HCI papers mention SD (e.g., see [27] for the numbers of SD at CHI conference), and we have observed that few researchers publish across both research communities.

Clarifying the inchoate relationship between UXD and SD could produce several benefits. First, it could identify opportunity areas where each practice might learn from and adopt best practices from one another. Second, it could drive the development of new tools that improve UXD and SD interaction and coordination. Third, it could inform design education, detailing what students need to learn in order to work effectively as a service or UX designer. Finally, it could help define where UXD and SD should be found and taught within academia; design schools, business schools, computer science schools, information systems schools, or somewhere else. The research and development of education, tools, and the disciplinary home for UXD and SD should start with a thorough investigation of the intersection of UXD and SD both in industry and academia. The goal of this paper is to help kickstart this process, to lay some ground work for the above developments by clarifying the prevailing understanding of the relation between UXD and SD. 
Our main research question asks: How do UX and SD communities understand the overlaps and the boundaries between these practices? We deployed an online survey to collect the opinions of UXD and SD professionals both in industry and academia. Specifically, the survey queried participants on (i) the scope of UXD and SD work, (ii) the subsumptive relationship between UXD and SD, and (iii) the theoretical roots and use of methods across the two practices. The findings shed light on the three topics, which turned out controversial in several ways and thus suggest new research directions. Based on the survey, we propose a research agenda for clarifying the relation and practices at the intersection of UXD and SD.

\section{RELATED WORK}

HCI has a long history of researching practice and of innovating on the methods and processes used by practitioners. Recent work has looked at how knowledge transfers from research to practice and from practice to research. This work largely proposes new ways of understanding this transfer process with the hope of improving a situation that does not seem to be working very well (e.g., [9],[3]) Researchers largely used interviews and observations of practitioners to understand their actions and needs (e.g., [36],[11]); however, they also used surveys to broadly explore an emerging area such as UXD and artificial intelligence [5], the same approach we use in this work exploring UXD and SD. Much of the practitioner focused research aims to develop new tools (e.g., [24],[21]) and also new design methods (e.g., [32],[22]). Researchers have cautioned about a need to better understand the needs and mindsets of practitioners if our research community wants to develop methods practitioners will actually adopt [31].

One new area where both design researchers and HCI researchers have shown an interest in design practice investigates the emergence of SD and compares it to the HCI communities' use of UXD. This seems largely driven by the fact that UXD increasingly involves the design of services (not products) and also the design of systems, where designers need a more systemic perspective to effect the societal change they desire (e.g,, sustainability, social computing) [34],[38]. Much of this work focuses on the distinctly different origins of SD and UXD as critical to understanding their similarities and differences [13],[8],[38]. The precursors of both practices go back to technology development in the 1980s. SD emerged at the time when advances in communication technology help drive an industrial transition from manufacturing products to performing services [23],[29]. SD started in business schools. It emerged from several different areas including marketing, operations, and management who all needed to adapt to the growing service sector. Creative design joined these efforts more recently. UXD followed a similar almost parallel history. First, HCI emerged from advances in computing technology and its need for user interfaces. The transition of computing from labs to offices meant workers, experts in their work and not in the operation of computers, needed interfaces that were useful and usable [10]. HCI brought together computer science and psychology. Their need for HCI continued with advances that produced interactive consumer products. The need to make products that consumers would choose and enjoy using gave rise to UXD, bringing design into HCI.
Over the duration of its history, SD has maintained a focus on three things: (i) the service encounter: the touchpoints where customers engage with a service; (ii) co-creation of value: how services create opportunities for customers and services to each generate value for the other during the performance of a service at a touchpoint; and (iii) the socio-material arrangement of the service: the ecology of people, places, and things within which the service exists and functions [18]. UXD, in turn, has focused on understanding the needs and desires of user, the design of digital interfaces, and the means of envisioning and prototyping interfaces designed to address user needs and desires.

Interestingly, practitioners have started to adopt both UXD and SD in their work, and researchers have begun exploring the overlap between the two practices. In discussing their overlap, design researchers claimed that SD remains more "mysterious" to most designers [13]. This might be because there is less design scholarship investigating service design in comparison to design writing about UX. For example, UXD research has many venues for publishing research while SD has only a few. Penin highlights an important distinction that seems to make SD different from all other design practices: "What differentiates service design from all other forms of design is that [it] is primarily the design of people, rather than the design of things for people" ([26] p.1). Designing people involves designing their jobs and the scripts they follow when executing these jobs. Researchers have also described SD as more far reaching, seeing it cover almost all of industrial design's concerns for material artifacts and UXD's concern for digital interactions [13]. The advocates of SD claim that UXD has much to gain from adopting a service perspective, especially (i) a systemic perspective for addressing more stakeholders; (ii) the product-service system framework that supports both things and performances; and (iii) the idea of co-creation of value between services and customers as compared to UXD's more narrow focus on user value [8].

Finally, there has been a growing interest in the UXD practice community about SD. Venues where professional designers exchange ideas, such as the blogging service Medium.com, have many articles discussing the differences between UXD and SD, if there really are differences between UXD and SD, what UX designers should know to start bringing SD into their UX practice, and even how a UXD professional might recognize when they are in fact doing SD as a normal part of their current practice (e.g., [12],[16] $[15],[4],[30])$. There is a desire among practitioners to understand the boundaries between these two practices, to possibly expand a UXD practice to incorporate SD or make claims about doing SD, and to understand how these design practices can work more effectively together.

\section{METHODS}

We wanted to better understand how researchers and practitioners of UXD and SD in industry and academia view the similarities and differences between UX and SD. We chose to deploy a web-based questionnaire in order to rapidly get a broad set of opinions. Online surveys allow data to be collected efficiently and effectively with little to no impact from temporal and geographical constraints. However, we are aware of its limitations such as difficulty in asking follow-up questions. We adopted the non-probabilistic voluntary 
method [28] because there is no known source of information describing the number and geographical spread of UX and service designers. This choice has a limitation of not controlling for selfselection bias. We distributed the survey to relevant mailing lists, including the global Service Design Network, SIGCHI, some of its local chapters (e.g. Finland), and across the authors' personal networks of UX and service designers.

The selection of survey questions was based on the gaps we identified in scientific literature (e.g. [13],[26]), the authors' own research on UXD and SD practice (e.g. [27][28]), discussions with practitioners working in the intersection of UXD and SD [27], and on the conflicting perspectives revealed in practitioners' blog posts (e.g. [12],[15]). The format of the questions, a Likert statement followed by an open-ended question asking for a justification for the rating, was tested in two small-scale, international surveys. We found the combination of quantitative and qualitative data provided insightful findings. One caveat is that our survey is not aimed to be a validated measuring tool on UXD/SD characteristics or knowledge. Instead, the questions and answer options are formulated in order to elicit respondents' stances and statements on the prevailing views and concerns around UXD and SD that we distilled through the aforementioned sources.

The survey questions queried "To what extent do you agree with the following statements, and why?", so both quantitative and qualitative data was collected for each such question. The following statements were designed to answer our research question.

Q1: Which one of the following areas is closest to your expertise area? Service Design (including all types of touchpoints) / Service Design (digital touchpoints) / User Experience Design / Experience Design, wider than UX / Product/Industrial Design / Other

Q2: A typical scope of UX designers' work includes:

- Designing with end users

- Designing with multiple actors, including users, service providers, and intermediaries

- Designing the products or services people will use

- Designing the work people will do

- Designing how products or services generate revenue

Q3: A typical scope of Service designers' work includes: < the same set of items as above>

Q4: UX Design is part of Service Design, i.e., UX design projects are often smaller projects that fit within a larger Service Design Project.

Q5: Service Design is part of UX Design, i.e., Service design projects are often smaller projects that fit within a larger UX Design Project.

Q6: UX Design methods and Service Design methods share the same theoretical roots.

Q7: There is no difference between UX Design methods and Service Design methods, they are the same.

Q8: UX Design methods should be used more in Service Design.

Please list some UX Design methods or techniques that you recommend to be used in Service Design.

Which UX Design methods or techniques are NOT applicable to Service Design?

Q9: Service Design methods should be used more in UX Design. $<$ the same sub-questions for SD as for UXD>
We had 197 participants from 31 countries complete the survey, and almost $68 \%$ of the responses came from participants in Europe. Two plausible reasons for the high number of European participants could be that (i) SD began in Europe [29] and the impact of this root remains substantial in the sense that many of SD designers are active in Europe; (ii) personal networks of the survey's implementers could attract respondents from certain countries. 42 respondents self-reported having an Academic role and 152 claimed an Industrial role. 108 self-reported having expertise in UX and 182 in SD. Descriptive and inferential statistics were applied to analyze quantitative data.

The open-ended questions of the survey were analyzed using inductive content analysis [7]. The first author started the analysis by open coding a subset of the data. Key messages were highlighted and coded for each response, so that several comments and codes could emerge from one response. A set of 124 unique open codes was identified and further analyzed by the first, second, and third authors in a workshop where they categorized them to a higher abstraction level. This resulted to 20 final codes. After discussion, four dimensions were identified that characterized the relation of UXD and SD in this data set.

After the workshop, the coding scheme was used to analyze the rest of the data. The same three authors were assigned separate questions to analyze. Inter-rater reliability was tested with $20 \%$ of the data, and the coding scheme was clarified with descriptions and updated until the inter-rater reliability, Cohen's unweighted kappa, was above 0.6. In total, our qualitative data set included 1025 comments related to the survey questions.

\section{FINDINGS}

We first report the findings of the quantitative analysis of Likert statements. We then report the findings of the qualitative analysis based on the shared justifications.

\subsection{Scoping of UX and service designers' work: Q2 \& Q3}

Q2 and Q3 probed how respondents' scope UXD and SD work. We assumed that respondents, depending on their education/work experience, perceived the respective scopes of work differently. Findings displayed in Fig.1 suggest that respondents viewed UXD and SD as being the same in terms of working with end users and making artifacts that people interact with. Respondents viewed UXD and $\mathrm{SD}$ as differing in the other three aspects. To evaluate whether the differences were statistically significant, non-parametric Wilcoxon Signed Rank tests were performed. There are three significant differences in perceiving the scope of work for UX and service designers (Table 1).

Specifically, work was considered to be different in terms of designing with multiple actors, designing people's work, and designing how products/services generate revenue. Respondents associated this work much more with service designers than with UX designers. While respondents viewed both UXD and SD as working with end users, our findings indicated that service designers address a larger set of stakeholders.

Another significant difference was around designing how products/services create revenue. While most respondents seemed to 


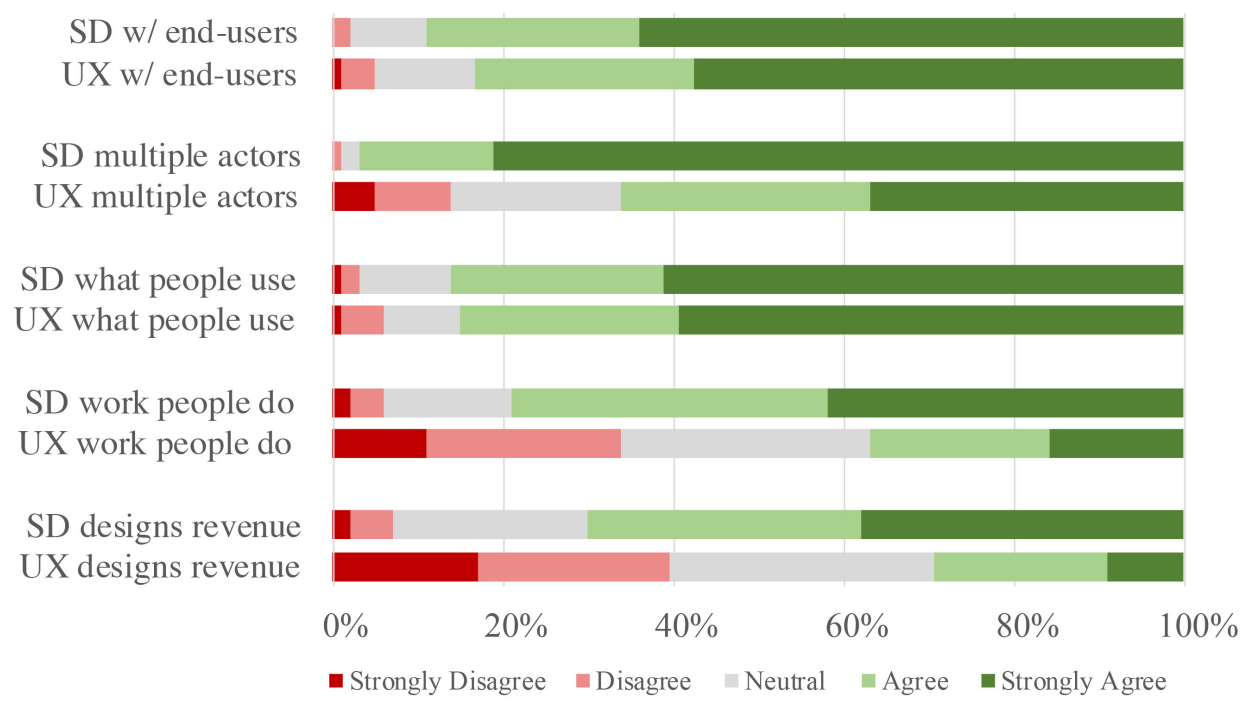

Figure 1: Descriptive statistics of agreement ratings on five UXD and SD aspects.

Table 1: Wilcoxon test results of ratings $(\mathrm{N}=197)$ for UXD Work (UXW) and SD Work (SDW).

\begin{tabular}{lllllll}
\hline & \multicolumn{2}{l}{ With Multiple Actors } & People's work & \multicolumn{2}{l}{ Revenue Generation } \\
\cline { 2 - 6 } & UXW & SDW & UXW & SDW & UXW & SDW \\
\hline Mean(SD) & $3.84(1.15)$ & $4.78(0.49)$ & $3.09(1.24)$ & $4.13(0.93)$ & $2.81(1.21)$ & $3.98(1.00)$ \\
Median & 4 & 5 & 3 & 4 & 9.37 & 4 \\
$Z$ & 8.43 & & 8.22 & & 0.000 \\
$p$ & 0.000 & & 0.000 & & \\
\hline
\end{tabular}

Table 2: Findings of Mann-Whitney U tests between GroupUX and GroupSD on Q2 (UXD work) ratings, Q3 (SD work) ratings, and the difference scores (Diff).

\begin{tabular}{|c|c|c|c|c|c|}
\hline & End Users & Multi Actors & Product/Service & People's Work & Revenue \\
\hline$U(\mathrm{UXW})$ & 3694 & 3282 & 3997 & 3076 & 2903 \\
\hline$Z$ (UXW) & 2.41 & 3.37 & 1.54 & 3.75 & 4.23 \\
\hline$p(\mathrm{UXW})$ & 0.016 & 0.001 & 0.124 & 0.000 & 0.000 \\
\hline$U(\mathrm{SDW})$ & 3850 & 3739 & 4380 & 3673 & 4118 \\
\hline$Z$ (SDW) & 1.69 & 2.55 & 0.02 & 2.05 & 0.76 \\
\hline$p(\mathrm{SDW})$ & 0.091 & 0.011 & 0.984 & 0.04 & 0.448 \\
\hline$U$ (Diff) & 3036 & 2828 & 4012 & 2680 & 2756 \\
\hline$Z$ (Diff) & 4.36 & 4.67 & 1.45 & 4.94 & 4.73 \\
\hline$p$ (Diff) & 0.000 & 0.000 & 0.148 & 0.000 & 0.000 \\
\hline
\end{tabular}

think neither role would carry the main responsibility for revenue generation, SD was seen as more connected to this responsibility.

We grouped respondents into two groups with respect to their self-reported expertise $(Q 1)$, GroupSD $(\mathrm{n}=82)$ vs. GroupUX $(\mathrm{n}=$ 110) (NB: the $1^{\text {st }} / 2^{\text {nd }}$ options for $S D$ and the $3^{\text {rd }} / 4^{\text {th }}$ options for UXD were combined for the respective groups. Five respondents did not specify their role clearly enough to be categorized). Mann Whitney tests were performed to verify if there were any significant differences between how GroupUX and GroupSD perceived the scope of UXD work (Q2) and SD work (Q3) (see Fig.2). For each respondent we derived a difference score (Diff block in Table 2) from their corresponding ratings for Q2 and Q3 to observe if the extent of change in perception of UXD work and SD work was significantly different between the two groups.

Findings (Table 2, the upper block) showed that there were highly significant differences between GroupUX and GroupSD in four of the five aspects (End Users, Multiple actors, People's work, Revenue) when referring to the typical scope of work for UX designers; 


\begin{tabular}{|c|c|c|c|}
\hline \multirow{3}{*}{$\begin{array}{r}\text { Designs with end } \\
\text { users }\end{array}$} & & GroupUX & GroupSD \\
\hline & UXW & 4.49 & 4.23 \\
\hline & SDW & 4.4 & 4.63 \\
\hline \multirow{2}{*}{$\begin{array}{l}\text { Designs with } \\
\text { multiple actors }\end{array}$} & UXW & 4.09 & 3.5 \\
\hline & SDW & 4.69 & 4.89 \\
\hline \multirow{2}{*}{$\begin{array}{r}\text { Designs the } \\
\text { product/services } \\
\text { people will use }\end{array}$} & UXW & 4.49 & 4.34 \\
\hline & SDW & 4.42 & 4.41 \\
\hline \multirow{2}{*}{$\begin{array}{l}\text { Designs the work } \\
\text { people will do }\end{array}$} & UXW & 3.35 & 2.7 \\
\hline & SDW & 4.02 & 4.3 \\
\hline \multirow{2}{*}{$\begin{array}{l}\text { Designs how the } \\
\text { product/service } \\
\text { generates revenue }\end{array}$} & UX & 3.11 & 2.36 \\
\hline & SDW & 3.94 & 4.05 \\
\hline
\end{tabular}

Figure 2: Mean agreement ratings on the five aspects of UXD and SD by two groups of respondents defined by expertise.

GroupUX tended to have a higher level of agreement on these aspects (Table 2). In contrast, the two groups differed significantly in only two aspects, when referring to the typical scope of work for service designers (Table 2, the middle block). GroupSD tended to have a higher level of agreement on these two aspects (Fig. 2; 4.89 vs. 4.69 for Multiple Actors; 4.3 vs. 4.02 for People's Work). For the difference score (Table 2, the lower block), four aspects (except Product/Service) had highly significant differences between the two groups. In fact, all respondents, irrespective of their expertise area, tended to agree that the five aspects of the scope of work were more applicable to service designers than to UX designers (Fig. 1), but respondents with SD expertise (GroupSD) perceived such a difference to be bigger than their counterparts with UXD expertise (GroupUX). These observations suggest that the expertise of respondents played a significant role in influencing their perceptions of the scope of UX and service designers' work.

\subsection{Subsumptive Relation between UXD and SD: Q4 \& Q5}

Q4 and Q5 probed how respondents viewed the relation between UXD and SD. Two contrasting views are that UXD is subsumed by SD or that SD is subsumed by UXD. We assumed that respondents' views would be shaped by their area of expertise.

As shown in Fig. 3, the majority of respondents tended to agree that UXD is a subset of SD. Interestingly, none with SD expertise agreed that SD is a subset of UXD (0\%). Respondents with industrial affiliation also had a very low level of agreement (3\% agree and $1 \%$ strongly agree) on this view. Findings of the Spearman's correlation test showed that there was a significant negative correlation $(\mathrm{N}=$ $\left.192, \mathrm{r}_{\mathrm{s}}=-.27, \mathrm{p}=000\right)$ between $Q 4$ and Q5. Logically, those who agreed that UXD is a subset of $\mathrm{SD}(Q 4 ; \mathrm{N}=194$, Mean $=3.33$, Std. Dev. $=1.27)$ tended to disagree that SD is a subset of UX D $(Q 5, \mathrm{~N}$ $=192$, Mean $=1.83$, Std. Dev. $=1.04$ ).

Results of Mann-Whitney tests showed that respondents with SD expertise tended to agree that UXD is a subset of SD $(\mathrm{N}=81$, Mean
$=3.7$, Std. Dev. $=1.21)$ whereas those with UXD expertise tended to remain neutral $(\mathrm{N}=108$; Mean $=3.06$, Std. Dev. $=1.22)$. The difference was highly significant $(U=3064, Z=3.62, p=0.000)$. Both groups tended to disagree with the view that SD is a subset of UXD. Those with SD expertise had a higher extent of disagreement $(\mathrm{N}=$ 80 , Mean $=1.42$, Std. Dev. $=0.67)$ than those with UXD expertise $(\mathrm{N}=107$, Mean $=2.16$, Std. Dev. $=1.16)$. The difference was highly significant $(\mathrm{U}=2695, \mathrm{Z}=4.68, \mathrm{p}=0.000)$.

Respondents with an Academic role tended to remain neutral that UXD is a subset of SD $(\mathrm{N}=42$, Mean $=3.02$, Std. Dev. $=1.26)$ whereas those with an Industrial role leaned towards agreement $(\mathrm{N}=152$, Mean $=3.41$, Std. Dev. $=1.26)$, but the difference was insignificant $(\mathrm{U}=2622, \mathrm{Z}=1.82, \mathrm{p}=.069)$. Both groups tended to disagree with the view that the SD is a subset of UXD; those with an Industrial role $(\mathrm{N}=150$, Mean $=1.69$, Std. Dev. $=0.91)$ had a higher extent of disagreement than those with an Academic role $(\mathrm{N}=42$, Mean $=2.36$, Std. Dev. $=1.26$ ), and the difference was significant $(\mathrm{U}=2186, \mathrm{Z}=3.28, \mathrm{p}=.001)$.

\subsection{Theoretical Roots and Methods of UXD/ SD: Q6 - Q7}

Q6 and Q7 asked whether respondents perceived that UXD and SD share the same theoretical roots, the same methods, and whether their expertise and affiliation played a significant role in influencing such perceptions.

Findings displayed in Fig. 4 suggest that the majority of respondents tended to consider that UXD and SD shared the same theoretical roots (68\% Agree or Strongly Agree), but they were more ambivalent with regard to the sameness of methods, albeit leaning towards disagreement (47\%, Disagree or Strongly Disagree). There was a highly significant correlation between the two views $(\mathrm{N}=193$, $\left.r_{s}=0.41, p=0.000\right)$. Logically, those who assumed that UXD and SD shared the same theoretical roots, the methods they employed should be more or less the same.

Results of Mann-Whitney tests showed no significant differences between the two groups of respondents with different expertise (Table 3$)$ in their views on the sameness of theoretical roots $(\mathrm{U}=$ $4332, \mathrm{Z}=0.03, \mathrm{p}=0.974)$ or the sameness of methods $(\mathrm{U}=3879, \mathrm{Z}$ $=1.404, \mathrm{p}=0.16)$ for UXD and SD. All respondents leaned towards the view that UXD and SD shared the same theoretical roots (mean > 3.0). They tended to disagree that UXD and SD used the same methods (mean < 3.0). The expertise of respondents did not play any significant role in shaping the view.

According to the Mann-Whitney tests, there was a significant difference between the two groups of respondents with different affiliation (Table 4$)$ in their views on the sameness of theoretical roots for $U X D$ and $S D(U=2374, Z=2.45, p=0.014)$. The differences in their views on the sameness of methods was insignificant $(\mathrm{U}=$ $3001 \mathrm{Z}=0.61, \mathrm{p}=0.543$ ). The affiliation of respondents played a role in shaping theoretical roots of UXD and SD.

\subsection{Method inclusion across UXD and SD: Q8 and Q9}

According to the statistical results for the sameness of methods (Q7) discussed in 4.3, the majority of respondents, irrespective of their expertise or affiliation, disagreed on method sameness in UXD 


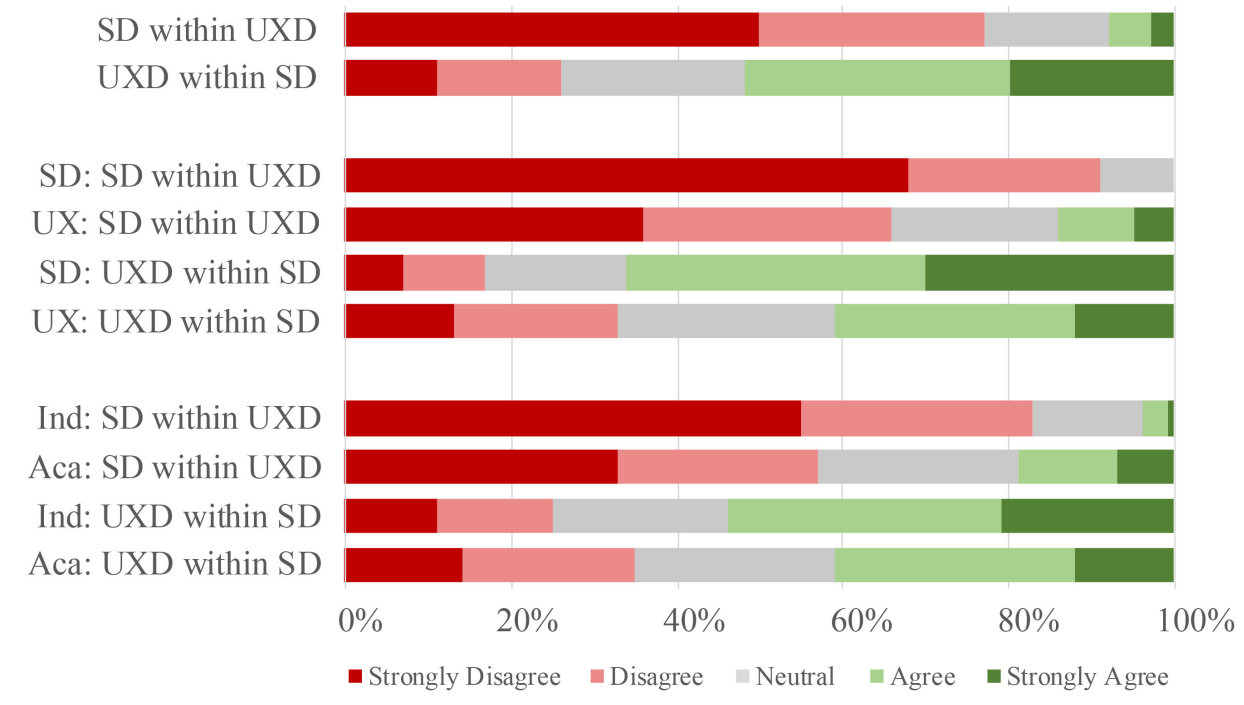

Figure 3: Distributions of agreement (\%) by All, grouped by Expertise (UXD vs. SD) and Affiliation (Industry vs. Academe) on the subsumptive relation between UXD and SD.

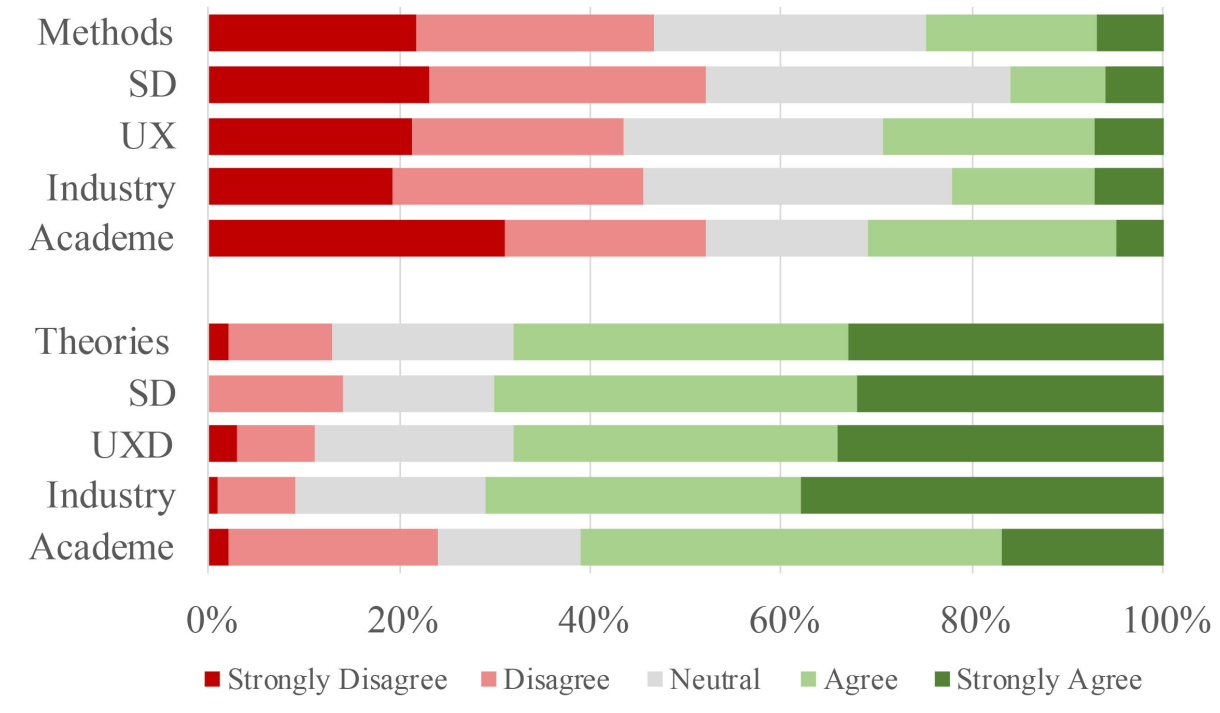

Figure 4: Distributions of agreement (\%) by All, grouped by Expertise (UXD vs. SD) and Affiliation (Industry vs. Academe) on the sameness of methods and theoretical roots between UXD and SD.

Table 3: Ratings on the sameness of theoretical roots and methods by respondent affiliation.

\begin{tabular}{llll}
\hline & Expertise & N & Mean (Std. Dev) \\
\hline Theoretical Roots & UX & 107 & $3.87(1.07)$ \\
Methods & SD & 81 & $3.89(1.01)$ \\
& UX & 107 & $2.70(1.22)$ \\
& SD & 82 & $2.46(1.13)$ \\
\hline
\end{tabular}


Table 4: Ratings on the sameness of theoretical roots and methods by respondent expertise.

\begin{tabular}{llll}
\hline & Affiliation & N & Mean (Std. Dev) \\
\hline Theoretical Roots & Academe & 41 & $3.51(1.09)$ \\
Methods & Industry & 152 & $3.97(1.01)$ \\
& Academe & 42 & $2.52(1.31)$ \\
& Industry & 152 & $2.65(1.16)$ \\
\hline
\end{tabular}

Table 5: Ratings on the method inclusion across UXD and SD by respondent expertise.

\begin{tabular}{llll}
\hline & Expertise & N & Mean (Std. Dev) \\
\hline UXD methods $\rightarrow$ SD methods & UX & 101 & $3.03(0.85)$ \\
& SD & 78 & $3.15(0.85)$ \\
SD methods $\rightarrow$ UXD methods & UX & 100 & $3.51(1.07)$ \\
& SD & 71 & $3.62(0.95)$ \\
\hline
\end{tabular}

Table 6: Ratings on the method inclusion across UXD and SD by respondent affiliation.

\begin{tabular}{llll}
\hline & Affiliation & N & Mean (Std. Dev) \\
\hline UXD methods $\rightarrow$ SD methods & Academe & 37 & $3.49(1.04)$ \\
SD methods $\rightarrow$ UXD methods & Industry & 147 & $3.14(0.82)$ \\
& Academe & 37 & $3.32(1.03)$ \\
\hline
\end{tabular}

and SD. These findings can serve as a backdrop against which responses to Q8 and Q9 on method inclusion across UXD and SD are interpreted.

There was a highly significant positive correlation between two views $\left(\mathrm{N}=171, \mathrm{r}_{\mathrm{s}}=0.23, \mathrm{p}=0.002\right)$. The majority of respondents (58\%; cf. 12\% Strongly Disagree or Disagree; 30\% Strongly Agree or Agree) were neutral on the view that UXD methods should be included more in SD methods $(\mathrm{N}=184$, Mean $=3.21$, Std. Dev $=0.87)$. They tended to agree (49\%; cf. $11 \%$ Strongly Disagree or Disagree; $40 \%$ Neutral) on the view that SD methods should be included more in UXD methods $(\mathrm{N}=176$, Mean $=3.52$, Std. Dev $=1.04)$. No significant correlation was found between the view that UXD methods should be included more in SD methods or the reverse.

No significant differences were found between the two groups of respondents with different expertise on the view of including more UXD methods in SD methods $(\mathrm{U}=3652, \mathrm{Z}=0.94, \mathrm{p}=0.347)$ or the reverse $(U=3348, Z=0.66, p=0.506)$ (Table 5). However, a significant difference was observed between two groups of respondents with different affiliation on the view of including more UXD methods in SD methods $(\mathrm{U}=2150, \mathrm{Z}=2.2, \mathrm{p}=0.027)$. Academic respondents tended to support this view more than their industrial counterparts, but their views were not significantly different for the reverse $(U=2210, Z=1.38, p=0.167)$ (Table 6).

\subsection{Qualitative Findings}

As explained in the Methods section, the open-ended "reasoning behind your rating" questions were analyzed through an inductive content analysis. This yielded 20 final codes. The most common one was 'SD more holistic than UXD' with 165 comments supporting this perspective among 1025 coded comments. The second most frequent code was 'Methods not boxed to a practice' (98 comments), which means that design methods can be used and mixed across UXD and SD practices, and are modified to best fit the problem context (Table 7).

The final codes were categorized into four high-level dimensions that characterize the main findings, revealing critical aspects about the relation between UXD and SD:

D1. User focus vs. Organization focus (253 comments)

- The focus of work can be on end users, or on value creation for service provider organizations.

D2. Single channel vs. Systemic view (318 comments)

- Focus on a single channel, e.g., a mobile application, versus a more holistic perspective to service journey orchestration and service system

D3. Ideal vs. Reality (113 comments)

- A contrast between what is considered ideal and what is the reality at work.

D4. Distinct vs. Indistinct (341 comments)

- UXD and SD are perceived as distinct from each other or overlapping.

From the four dimensions and 20 codes, we deduced five practiceoriented themes, offering more concrete grounds for understanding the current perceptions on boundaries and interplays between UXD and SD. The links among 20 codes, 4 dimensions and 5 themes are depicted in Figure 5 
Table 7: Number of comments coded under the dimensions where D1, D2, D3 and D4 are the four high-level dimensions

\begin{tabular}{llll}
\hline Dimension (D): Code(C) & Comments & Dimension: Code (continued) & Comments \\
\hline D2/C1: SD more holistic than UXD & 165 & D2/C11: Across touchpoints & 34 \\
D4/C2: Methods not boxed to a field & 98 & D2/C12: Digital & 32 \\
D4/C3: Co-existence with overlaps & 88 & D2/C13: Single touchpoint & 30 \\
D1/C4: User orientation & 75 & D2/C14: Product level & 30 \\
D4/C5: Field-specific competence & 69 & D3/C15: Should be encompassing & 28 \\
D3/C6: Depends on the project & 65 & D2/C16: User interface level & 27 \\
D4/C7: Co-existence as separate fields & 62 & D4/C17: Division of fields not meaningful & 24 \\
D1/C8: Stakeholder orientation & 50 & D1/C18: Business orientation: strategy & 24 \\
D1/C9: UX as a goal for all designs & 46 & D1/C19: Business orientation: revenue & 23 \\
D1/C10: Revenue not the main focus & 35 & D3/C20: Constraint: Organizational factors & 20 \\
& & Total & $\mathbf{1 0 2 5}$ \\
\hline
\end{tabular}

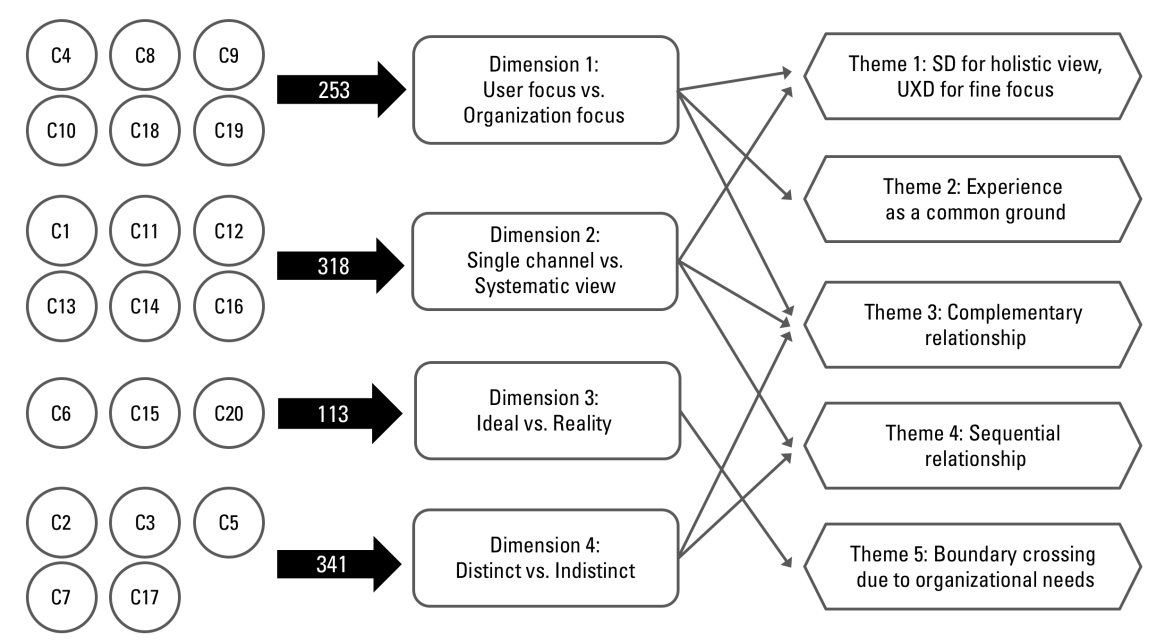

Figure 5: Links between clusters of codes (C) with the total numbers of comments, four dimensions and five themes

4.5.1 Theme 1: SD for a holistic view, UXD for fine focus. Resonating with our quantitative analysis, one of the most frequently appearing comments was SD is more holistic than UXD. The respondents explained the reasons in terms of the focus of the work (as reflected in Dimension 1. User focus vs. Organization focus) and the object of design (as reflected in Dimension 2. Single channel vs. Systemic view). While UXD focuses on interaction between a user and a single artefact, typically digital, SD takes into consideration the "orchestration" (SD researcher, respondent ID - referred to as ID hereinafter - 72) of different artefacts across a user's journey to achieve his or her goal. In that journey and process, multiple stakeholders are involved and contribute to value co-creation. "Services involve multiple actors who contribute directly or indirectly, synchronously or asynchronously in the value co-creation. This perspective would drive the need to get an understanding of the attributes and relationship among the multiple players, in turn visualising their individual contribution to value co-creation." (SD practitioner, ID 47).

Many respondents mentioned that saying, "UXD is part of SD" does not imply the SD subsumes UXD. They suggested this had more to do with the breadth (SD being broader) and the depth (UXD being deeper) of the practice. While SD provides a holistic perspective with an understanding of an ecosystem and its impact on business, UXD goes deeper in detail in the analysis and development of a specific interaction. One of the respondents called it "zooming in \& out". "Service design has a holistic view of customer experiences/journeys. A customer experience can include one or several user experiences with digital touchpoints. So, if we "zoom in" on a digital touchpoint of a service, we get a user experience that needs to be designed in detail by a UX (and perhaps a UI) designer." (SD student, ID 120). "...the UX perspective is narrower (user perspective) than SD perspective (also includes business and staff), but they are more detailed. Where for us $S D$ is mainly concerned about how everything works together, UX design goes further in detailing the actual product." (SD practitioner, ID 97).

4.5.2 Theme 2: Experience as common ground. What was highlighted as "common ground" or a shared principle between the two practices was the notion of 'experience', either user experience, customer experience, or stakeholder experience. "UX and Service 
design fall under Experience Design.” (UXD practitioner, ID 176). "UX design as a term is becoming obsolete, Experience Design includes $u x$, $c x$ and service in traditional sense of the definitions." (SD practitioner, ID 189)

Our quantitative analysis showed respondents' tendency to agree that UXD and SD share the same theoretical roots. This can be attributed to the fact that both draw from theories of design thinking and both view themselves as part of Human-Centered Design (HCD), centering around experience as a core principle. "Design thinking, inclusivity and an understanding of a user are at both practices roots" (SD practitioner, ID 84). "HCD or UCD. Same principles, only that SD focuses on the big picture" (SD teacher and practitioner, ID 76)

We also found that the terms, UXD and Experience Design, are still elastic, and this influences the varying perceptions on the subsumptive relationship between UXD and SD. Those who think that the term User Experience Design equals Experience Design view that SD is part of UXD. "...using UX as an umbrella term and service design and interaction design as disciplines within. We all make user experiences." (SD practitioner, ID 85). Some respondents, on the other hand, define Experience Design as an umbrella concept under which both UXD and SD fall (as exemplified in the abovementioned comment by ID 176). In this view, UXD focuses on digital UI design and Experience Design refers to design for experiences in a broader sense.

4.5.3 Theme 3: Complementary relationship: By adopting methods. 341 instances from the respondents' comments were coded under Dimension 4. Distinct vs. Indistinct. Diverse views under this dimension were observed in aspects of field-specific competence, commonality and differences in methods, and a shared goal.

A set of field-specific expertise was highlighted regarding the distinction between the two practices. The respondents explained how the expertise of one practice can complement another. Prototyping, user testing, and implementation were mentioned as UXD expertise, which could be integrated more in SD. In other words, prototyping and testing are recognized as less developed areas in $\mathrm{SD}$, possibly due to the nature of service being co-produced in a real context involving multiple actors and multiple channels. "In certain cases, having a more accurate or focused way of doing user testing for example can be beneficial [for Service Design]." (SD practitioner, ID 50). "More specialized expertise needs to be involved as services are piloted/implemented." (SD practitioner, ID 54)

In addition, various kinds of user research methods, such as ethnographic observations, contextual inquiry, and personas were mentioned as what can bring more user-centeredness to SD, preventing service design projects from overly focusing on business strategies and helping them find harmony between business- and user-orientation. "It is easy to forget the role of the user in value proposition and in experiments, to find the right amount of hard facts to support one direction over the other, this is a risk in service design." (UXD practitioner, ID 152)

On the other hand, a holistic and systemic thinking is regarded as SD expertise, which can help to broaden UX designers' perspectives. The methods helping to position UXD in a bigger picture were recommended for UXD, such as customer journey mapping for understanding the roles of individual touchpoints in a larger journey, service blueprinting for helping UXD identify UX problems from the backstage operations, and stakeholder mapping for making sense of collaborative networks. "UX designers generally lack the view of the ecosystem of things, and focus their attention to just design the product right; not necessary the right product in the landscape." (UXD practitioner, ID 20). "Service design widens the scope of $u x$, and by including several stakeholders, and different needs it helps to paint a clearer picture of which interactions can benefit who." (SD practitioner, ID 84)

4.5.4 Theme 4: Sequential relationship: SD provides a strategy for $U X D$. Several respondents mentioned that SD develops a strategy as an output of its design process. Some noted this could lead to a UXD brief. This view was shared by both UXD and SD respondents. In this view, SD comes early in the project, sets business strategies, and identifies the channel or touchpoint where design is needed. These can be starting points for a design brief that guides UXD work. "...UX is the execution of a proposition/strategy - which SD works on." (SD teacher \& practitioner, ID76). "The project should start from the service and overall structure and design, then scale down. Start high level and broad with the service then the details of the touchpoints." (UXD practitioner, ID 35). "I've always thought that Service Design may be a more strategic approach than UX. In that sense findings in Service Design project may lead to a UX design brief but sometimes SD leads to design of physical services." (UXD practitioner, ID 155)

4.5.5 Theme 5: Boundary crossing due to organizational needs. In respondents' comments, 116 instances were coded under Dimension 3. Ideal vs. Reality. Many respondents shared that what defines the work scope in reality is the organizational needs and legacy than a scholarly definition. "At the end of the day, I think it depends on the organization and its needs. These can be mutually exclusive domains-or they can be part of each other. It's less about the industry aligning on a definition than companies having a clear picture of their needs and aligning (and stacking) their design team to match. (SD practitioner, ID 95)

The majority of comments originated from Q2 regarding the scope of UX designer's work, which indicates that UX designers' job is not always what people would expect or like it to be. For example, while user focus was a common theme in UXD work (Fig 1), user-centered design approach may easily twist to user interface or user retention design. This explains why a UX designer seems not to work with the users any more than a service designer (Fig 1). "People should and want, but seldom have the full opportunity to work with end users, stakeholders and other such entities. . . mostly UX designers do only 'UI design' sort of things." (SD practitioner, ID 55). "Ideally we should just care about the users and design for them. If the design fits a gap, the revenue just happens. But in the real world, we work for a salary for a for-profit company and the value proposition of the ux team extends to maintaining the user base regardless of the actual user needs." (UXD practitioner, ID 38)

In real life, the constraints and needs of organizations and projects vary, which requires adjustments to the work scopes and considerations. For example, innovation requires a broader perspective than usability fixes. "I have come across contexts where stakeholder's perception of their service is quite limited and they believe a 'new design' for the website or simply a website/ service portal 
will solve all the issues. Often there is far more involved than that and getting a whizzy looking UI simply creates more problems than it solves." (UXD practitioner, ID 159)

Our data indicates the scope of UXD work is, or should be, expanding and UX designers need to have a wider set of skills than is typically part of user-centered design. The scope of UXD work seems to be adjusted to the contextual needs, and what is ideal in one context may be unwanted or even utopian in another. "In real life settings, being profitable is very important for long-term development of an organisation. I do not encourage utopian designer ideal that experience is the only consideration." (UXD researcher, ID 137)

\section{DISCUSSION}

The overall goal of this research is to investigate the ongoing transformation of design practice and to pass this knowledge on to UX and HCI researchers. In this paper, our focus is on understanding the shifting overlaps and boundaries between UXD and SD practices. In the previous section, we reported the findings of our international online survey, where 197 practitioners and researchers participated. Below, we discuss the implications of our findings, addressing each aspect of our research question, i.e. the scope of designer's work, subsumptive relationship, and theoretical roots and use of methods. For each of these, we propose topics for a research agenda to address the controversial findings of our survey and to maintain the relevance of UX research in the changing practices.

\subsection{The scope of UXD work in creating services}

UXD and SD respondents agreed that both practices have a strong concern for the end user's experience in their work. They also agreed that SD takes a more holistic view, cares about a broader set of actors than just the user, focuses on how the service generates revenue, and designs the actions of people in addition to the design of things. Respondents shared that the content of UXD work is different; however, the scope of UXD seemed less clear than the scope of SD.

The project being worked on seemed to be the strongest driver to define the work scope, regardless of whether someone viewed themselves as UX or service designers. We suspect the increasing use of digital interfaces for services and the transition of the tech industry towards services and service platforms caused designers to find themselves increasingly taking on the work of the other practice. Work in small companies and small design consultancies may also contribute to such cross-boundary practice. Collectively, this is likely what causes job ads to sometimes be indistinguishable for UX or service designers. There does seem to be a trend of UX designers rebranding themselves as service designers [12], even if their practice would stay in UX design.

As reviewed in the related work section, UXD has been continually expanding its scope along with emerging technologies and social agenda [8],[38]. Our survey findings showed a high variation in the scope of UXD work, which indicates the need to clarify the scope of UXD work in the current trend of making digital services or digital channels for brick and mortar services. A few recent papers forecast possible expansions of UX design and research by embracing service thinking (e.g. [37][38]), yet little has been done to look into and clarify actual changes of UXD work. We therefore propose this as the first topic on our research agenda. This can be done, for instance, by studying job descriptions (c.f. a similar research conducted on graphic design [6], or projects where separate people work on SD and UXD. The second topic to add to the research agenda is to conduct longitudinal research on the development of the scope of both UXD and SD work over time, i.e., whether the two practices are integrating or differentiating. The new job role of Business Designer could be included in this investigation.

\subsection{Towards synergetic interplay}

The quantitative findings showed high inconsistencies between SD respondents and UXD respondents on subsumptive relationship between the two practices. None of the SD respondents viewed SD as a subset of UXD. While most UXD respondents agreed with this, some did view SD as a subset of UXD. It is surprising that more academics viewed SD as a subset of UXD. This might be somewhat explained by the fact that many design schools that have taught UXD for many years are now offering courses to their UXD students on SD. This may have more to do with hierarchies within an academic department than it does with design practice.

The framing of one practice subsuming another creates a hierarchical relationship, and that likely is what triggers the conflicting views. Interestingly, some respondents proposed that the relationship might best be viewed as sequential, rather than hierarchical. They noted that the holistic, systemic perspective of SD, with its concern for many different actors worked well in support of creating and delivering a design strategy. And the strategy could be the seed of one or more design briefs that might drive the detailed and iterative work of UXD, often focused on the needs of users at a single touchpoint or channel within a service. This sequential interplay between SD and UXD might have been experienced by practitioners sporadically, as hinted at by the survey respondents and [12]. However, more research efforts should be made to account for the phenomenon and strategise for a synergetic interplay. For example, in which phases of the project should SD practice and UXD practice be allocated? For the two practices work sequentially, how to manage accountability between the two? In which format and what kind of process? How should the value co-creation model of the project be informed to UXD work, and how should the end user experience be informed to SD work? Some existing work discuss how the upper level value creation model could inform the design of service encounter interactions (e.g. see [24]), but they focus on the customer-company interaction from a marketing strategy perspective. We propose as the third topic on the research agenda to explore ways for UXD and SD to work interdependently in a project. This research agenda may involve case studies and development of new processes and tools.

This finding also has interesting implications for education. Service design classes might focus on producing UX design briefs as a deliverable along with a strategy, and UXD classes might use service strategies and design briefs as a starting point for a studio project. Interdisciplinary UXD classes often mix designers, software developers, and behavioral scientists working together. It could be interesting to add in service designers and business students to this mix. Investigating approaches to designing education of practitioners 
with expertise covering both UXD and $S D$ is the fourth item on our research agenda.

\subsection{Appropriation of methods}

As researchers we are surprised about the high level of agreement on UXD and SD sharing the same theoretical roots. Value co-creation in SD and usability engineering in UXD seem far apart. Respondents specified the theories they viewed as similar to design thinking, which seems to form a new theoretical basis to the ongoing transformation. From this perspective, UXD and SD do share common theories around design as a mode of knowledge production. Since both UX and service designers focus on design, they likely view each other as much more similar than they see themselves in comparison to their main collaborators, e.g., software engineers, usability experts, or data scientists for UX designers, and business consultants for service designers.

On the contrary to the perception on theoretical roots, only $25 \%$ think UXD and SD use the same methods. Each practice appeared to have a list of its 'signature' methods (e.g. usability testing for UXD and service blueprint for SD). While differences in methods were recognized, respondents frequently commented that methods are not tied to a design practice. Both UX and service designers are flexibly adopting methods from each other's practice and appropriating them to the given project circumstances. This finding confirms the view that practitioners adapt their methods contextually across the boundaries [35],[19],[2]. Further research into how UXD and SD methods are adopted across the boundaries and appropriated to various project requirements will deepen our understanding on actual perceptions and practices of methods. As Boehner et al [2] demonstrated, studying how methods are appropriated in UXD and SD projects will help to clarify varying scopes and foci of the two practices, as well as commonalities. We propose this as the last item in our research agenda.

\section{CONCLUSIONS}

We conducted an international online survey $(n=197)$ to investigate how practitioners and researchers in UXD and SD perceive the overlaps and boundaries of the two practices. The responses provide a valuable new perspective to the unfolding relationship between the two. Our findings show points of agreement, points of contention, surprising perspectives on theory and methods, and a fluidity across the practice where designers from both practices appropriate each other's methods and concerns to fulfill the needs of a given project. We believe that such clarifications of the current perceptions and (dis)agreements will be the stepping stone to further explore distinctive roles of each practice and synergetic opportunities from their interplay, which can contribute to the transition of the design object in HCI. The analysis of the findings directed us to identify a set of future research topics aiming to inform both practice and education. We call for more research on

- Clarity on the currently blurry scope of UXD work

- The development of the scope of both UXD and SD work over time

- Ways for UXD and SD to work interdependently in a project

- Approaches to designing education of practitioners with expertise covering both UXD and SD
- Adoption of methods across the boundaries of UXD and SD and appropriation of them according to project requirements

This survey reveals important characteristics of the UXD and SD practice at a time when the cohabitation practices of the two design approaches are still forming. More studies on the roles and interplay of UXD and SD in real-life projects are needed to alleviate the confusions found in this study. We hope this knowledge can eventually lead to more success stories of research that has an impact on design practice. Then, it would be intriguing to replicate this survey and see how understanding of the overlaps and boundaries between UXD and SD has evolved and how research has been able to influence the practice in this area.

\section{ACKNOWLEDGMENTS}

We thank the survey participants and the participants in our workshops at CHI'18 and Nordes'19 for making this study possible. The first author was supported by Business Finland, grant 81/31/2020, and the second author by the Singapore Ministry of Education Academic Research Fund (R-290-000-012-114).

\section{REFERENCES}

[1] Steve Benford, Gabriela Giannachi, Boriana Koleva, and Tom Rodden. 2009. From interaction to trajectories: designing coherent journeys through user experiences. In Proceedings of the SIGCHI Conference on Human Factors in Computing Systems. ACM, New York, 709-718. https://doi.org/10.1145/1518701.1518812

[2] Kirsten Boehner, Janet Vertesi, Phoebe Sengers, and Paul Dourish. 2007. How HCI interprets the Probes. In Proceedings of the SIGCHI Conference on Human Factors in Computing Systems. ACM, New York, 1077-1086. https://doi.org/10. $1145 / 1240624.1240789$

[3] Lucas Colusso, Ridley Jones, Sean A. Munson, and Gary Hsieh. 2019. A Translational Science Model for HCI. In Proceedings of the 2019 CHI Conference on Human Factors in Computing Systems. ACM, New York. https://doi.org/10.1145/3290605. 3300231

[4] Designlab. 2018. Service Design and UX Design: What's the Difference? Retrieved Feb 8, 2021 from https://blog.prototypr.io/service-design-and-ux-design-whatsthe-difference-508c41c65217

[5] Graham Dove, Kim Halskov, Jodi Forlizzi, and John Zimmerman. 2017. UX design innovation: Challenges for working with machine learning as a design material. In Proceedings of the 2017 chi conference on human factors in computing systems. ACM, New York, 278-288. https://doi.org/10.1145/3025453.3025739

[6] Paulo Roberto Nicoletti Dziobczenski and Oscar Person. 2017. Graphic designer wanted: A document analysis of the described skill set of graphic designers in job advertisements from the United Kingdom. International fournal of Design 11, 2, 41-55.

[7] Satu Elo and Helvi Kyngäs. 2008. The qualitative content analysis process. fournal of Advanced Nursing 62, 4, 107-115.

[8] Jodi Forlizzi and John Zimmerman. 2013. Promoting service design as a core practice in interaction design. In Proceedings of the 5th IASDR World Conference on Design Research. IASDR Press.

[9] Colin M. Gray, Erik Stolterman, and Martin A. Siegel. 2014. Reprioritizing the relationship between $\mathrm{HCI}$ research and practice: bubble-up and trickle-down effects. Proceedings of the 2014 conference on Designing interactive systems. ACM, New York, 725-734. https://doi.org/10.1145/2598510.2598595

[10] Jonathan Grudin. 1991. Interactive Systems: Bridging the gaps between developers and users. Computer 24, 4 (April, 1991), 59-69. https:// 10.1109/2.76263

[11] Scarlett R.Herring, Chia-Chen Chang, Jesse Krantzler, and Brian P. Bailey. 2009. Getting inspired! Understanding how and why examples are used in creative design practice. In Proceedings of the SIGCHI Conference on Human Factors in Computing Systems. ACM, New York, 87-96. https://doi.org/10.1145/1518701. 1518717

[12] Ben Holliday. 2017. When is User Experience (UX) and Service Design the same thing? Retrieved Feb 8, 2021 from https://medium.theuxblog.com/when-is-userexperience-ux-and-service-design-the-same-thing-c739c3773bf5

[13] Stefan Holmlid. 2007. Interaction design and service design: Expanding a comparison of design disciplines. In Proceedings of the 2nd Nordic Design Research Design Conference.

[14] Ohad Inbar and Noam Tractinsky. 2012. Lowering the line of visibility: incidental users in service encounters. Behaviour \& Information Technology 31, 3, 245-260. 
[15] Anish Joshi. 2019. When does UX Design become Service Design? Retrieved Feb 8,2021 from https://medium.com/search?q=When\%20does\%20UX\%20Design\% 20become\%20Service\%20Design $\% 3 \mathrm{~F}$

[16] Justinmind. A primer on service design for UX designers. 2018. Retrieved Feb 8 2021 from https://uxplanet.org/a-primer-on-service-design-for-ux-designers$7696 \mathrm{~d} 2498 \mathrm{bc0}$

[17] Victoria Karaseva and Ahmed Seffah. 2015. The human side of software as a service: building a tighter fit between human experiences and SOA design practices. In proceedings of the Eighth International Workshop on Cooperative and Human Aspects of Software Engineering. IEEE, 105-108. https://doi.org/10.1109/CHASE 2015.31

[18] Lucy Kimbell and Jeanette Blomberg. 2017. The object of service design. In Designing for Service: Key Issues and New Directions, Daniela Sangiorgi and Alison Prendiville (eds.). Bloomsbury Publishing, London, UK, 81-94.

[19] Jung-Joo Lee. 2014. The True Benefits of Designing Design Methods, Artifact: Journal of Design Practice. 3, 2, 5.1-5.12.

[20] Jung-Joo Lee. 2020. Service design and blind mice. Interactions, 27, 2, 20-21.

[21] James Lin, Mark W. Newman, Jason I. Hong, and James A. Landay. 2000. DENIM finding a tighter fit between tools and practice for Web site design." In Proceedings of the SIGCHI conference on Human Factors in Computing Systems. ACM, New York, 510-517. https://doi.org/10.1145/332040.332486

[22] Sara Ljungblad, and Lars Erik Holmquist. 2007. Transfer scenarios: grounding innovation with marginal practices." In Proceedings of the SIGCHI conference on Human factors in computing systems. ACM, New York, 737-746. https://doi.org/ $10.1145 / 1240624.1240738$

[23] Matthew L. Meuter, Amy L. Ostrom, Robert I. Roundtree, and Mary Jo Bitner. 2000 Self-service technologies: understanding customer satisfaction with technologybased service encounters. fournal of Marketing 64, 3, 50-64.

[24] Roshanak Zilouchian Moghaddam, Brian P. Bailey, and Christina Poon. 2011 Ideatracker: an interactive visualization supporting collaboration and consensus building in online interface design discussions." In IFIP Conference on HumanComputer Interaction. Springer, Berlin, Heidelberg, 259-276

[25] Lia Patricio, Raymond P. Fisk, Joao F. Cunha, and Larry Constantine. 2011. Multilevel service design: From customer value constellation to service experience blueprinting. Journal of Service Research 14, 2, 180-200.

[26] Lara Penin and Cameron Tonkinwise. 2009. The Politics and theatre of service design. In Proceedings of the Conference of the International Association of Societies of Design Research. IASDR Press 4327-4338.

[27] Virpi Roto, Jung-Joo Lee, Tuuli Mattelmäki, and John Zimmerman. 2018. Experience Design meets Service Design: Method Clash or Marriage? In Extended Abstracts of the 2018 CHI Conference on Human Factors in Computing Systems. ACM, New York. https://doi.org/10.1145/3170427.3170626
[28] Virpi Roto, Val Mitchell, Stuart Cockbill, Jung-Joo Lee, Efe L-C Law, and John Zimmerman. 2021. Introduction to Service Design for UX Designers. In CHI Conference on Human Factors in Computing Systems Extended Abstracts (CHI '21 Extended Abstracts), May 08-13, 2021, Yokohama, Japan. ACM, New York, NY, USA

[29] William Schofield. 1996. Survey sampling. In Data Collection and Analysis, Roger Sapsford and Victor Jupp (eds.). SAGE: London, 25-56.

[30] Lynn G. Shostack. 1982. How to design a service. European fournal of Marketing 16, 1, 49-63.

[31] Emilie van Spronsen. 2019. Touchpoint Strategy Canvas - a practical tool for combining service design with UX design. Retrieved Feb 8, 2021 from https://medium.com/search?q=Touchpoint $\% 20$ Strategy $\% 20$ Canvas $\% 20---$ $\% 20$ a\% 20 practical $\% 20$ tool $\% 20$ for $\% 20$ combining $\% 20$ service $\% 20$ design $\% 20$ with\% 20UX\%20design

[32] Erik Stolterman. 2008. The nature of design practice and implications for interaction design research. International fournal of Design 2, 1.

[33] Petra Sundström, Elsa Vaara, Jordi Solsona, Niklas Wirström, Marcus Lundén, Jarmo Laaksolhati, Annika Waern, and Kristina Höök. 2011. Experiential artifacts as a design method for somaesthetic service development. In Proceedings of the 2011 ACM symposium on The role of design in UbiComp research \& practice. ACM, New York, 33-36. https://doi.org/10.1145/2030031.2030041

[34] Michael Thompson. 2018. The CX tower of Babel: what CX job descriptions tell us about corporate CX initiatives. Interactions 25, 3, 74.

[35] Karim Touloum, Djilali Idoughi, and Ahmed Seffah. 2018. Adding UX in the Service Design Loop: The Case of Crisis Management Services. Interaction Design and Architecture(s) fournal. 37, 47-77.

[36] Alan Woolrych, Kasper Hornbæk, Erik Frøkjær, and Gilbert Cockton. 2011. Ingredients and Meals Rather Than Recipes: A Proposal for Research That Does Not Treat Usability Evaluation Methods as Indivisible Wholes. International fournal of Human-Computer Interaction. 27, 10, 940-970.

[37] Qian Yang, Alex Scuito, John Zimmerman, Jodi Forlizzi, and Aaron Steinfeld. 2018. Investigating how experienced UX designers effectively work with machine learning. In Proceedings of the 2018 Designing Interactive Systems Conference. ACM, New York, 585-596. https://doi.org/10.1145/3196709.3196730

[38] Daisy Yoo, Anya Ernest, Sofia Serholt, Eva Eriksson, and Peter Dalsgaard. 2019. Service Design in HCI Research: The Extended Value Co-creation Model. In Proceedings of the Halfway to the Future Symposium (HTTF 2019), November 19-20, 2019, Nottingham, United Kingdom. ACM, New York. https://doi.org/10. $1145 / 3363384.3363401$

[39] John Zimmerman and Jodi Forlizzi. 2019. Service Design. In Encyclopedia of Human-Computer Interaction ( $2^{\text {nd }}$. ed.). Interaction Design Foundation. Retrieved August 27, 2020 from https://www.interaction-design.org/literature/book/theencyclopedia-of-human-computer-interaction-2nd-ed/service-design 